\title{
Probing two-particle exchange processes in two-mode Bose-Einstein condensates
}

\author{
Luis Benet, ${ }^{1, \text { f Diego Espitia, }{ }^{1} \text { and Daniel Sahagún }}{ }^{2}$ \\ ${ }^{1}$ Instituto de Ciencias Fúsicas, Universidad Nacional Autónoma de México (UNAM) \\ Apdo. Postal 48-3, 62251 Cuernavaca, México \\ ${ }^{2}$ Instituto de Física, Universidad Nacional Autónoma de México (UNAM) \\ Apartado Postal 20-364, 01000 Cd. Mx., México
}

(Dated: October 14, 2018)

\begin{abstract}
We study the fidelity decay and its freeze for an initial coherent state of two-mode Bose-Einstein condensates in the Fock regime considering a Bose-Hubbard model that includes two-particle tunneling terms. By using linear-response theory we find scaling properties of the fidelity as a function of the particle number that prove the existence of two-particle mode-exchange when a non-degeneracy condition is fulfilled. Tuning the energy difference of the two modes serves to distinguish the presence of two-particle mode-exchange terms through the appearance of certain singularities. We present numerical calculations that illustrate our findings, and propose exploiting a Feshbach resonance to verify experimentally our predictions.
\end{abstract}

PACS numbers: 03.75.Lm, 03.75.Kk, 05.30.Jp, 03.75.Dg

\section{INTRODUCTION}

The Bose-Hubbard model became a workhorse to describe interactions of ultracold bosonic gases trapped by neighboring potentials after its striking success with the Mott insulator-superfluid transition [1, 2]. Its most simple physical realization, when only two bosonic states can be occupied [3, is experimentally obtained by trapping the condensate in a double-well potential 4. This system is interesting because it is the simplest scheme for atom interferometry. In addition to interference phenomena [5, 6], it also exhibits quantum tunneling and self trapping effects [7, 8] such as Josephson oscillations 9. It has even been used to produce and study many-particle entanglement 10 and dynamically generate spin-squeezed states [11. Alternative methods to optical lattices have been demonstrated by splitting a single-component Bose-Einstein condensate (BEC) on atom chips either with pure DC magnetic fields [12] or by dressing static fields with RF potentials $13-15$ as proposed in [16. Understanding the effects originated on inter-atomic collisions has already been exploited to overpass the classical limit in atom interferometers [6], for example. Here we employ an extended Bose-Hubbard Hamiltonian to increase the possibilities in this direction.

Several interchange terms arise in the Bose-Hubbard model in a double-well potential where only the lowest level in each well is populated and the corresponding wave functions have a small overlap [4, 17. In particular, two terms accounting for two-particle modeexchange processes appear in the derivation of the Hamiltonian. These terms are often neglected assuming that two-particle processes are rare for diluted ultracold gases. Yet, Ref. [17] points out that there is a better agreement with the experimental results when these processes are

*Electronic address: benet@fis.unam.mx included. In this paper we probe the relevance of these terms by studying dynamical properties linked with twoparticle tunneling processes. We consider the dynamical stability of the quantum evolution under small system perturbations for the two-mode Bose-Hubbard model using the fidelity or Loschmidt echo $18-20$, whose decay has been studied for different parameter ranges and types of perturbations in the Bose-Hubbard model [21 23].

Prosen and Žnidarič noticed that fidelity stops decaying, staying essentially constant (modulo some oscillations) for relatively long times whenever the timeaveraged expectation value of the perturbation vanishes 24. This phenomenon is called fidelity freeze. It was later shown that symmetries can also induce this behavior if the diagonal matrix elements of the perturbation vanish, e.g. when the perturbation is not invariant under the time-reversal symmetry [25]. Note that the freeze of fidelity was actually observed in simulations for bosonic and fermionic many-body systems 22, 26, but it was attributed to the non-linearities introduced by the interactions between the particles. Our purpose is to draw attention to this phenomenon and exploit it, within the context of the Bose-Hubbard model.

The paper is organized as follows. In Sect. III from a generalized two-mode Bose-Hubbard Hamiltonian we derive a the fidelity freeze $F_{F r}$ associated to an initial macroscopic trial state [27. In Sect. III we show analytically and numerically that the scaling properties of the fidelity freeze display a transition in terms of the number of particles if the interaction includes two-particle modeexchange terms. In addition, when certain degeneracy condition is fulfilled by tuning the energy difference of the two levels, the fidelity freeze tends abruptly to zero. This yields insight into many-body tunneling processes and provides a method to calibrate the system to enhance the fidelity freeze. In Sect. [V] we summarize our results and address the possibility to access the parameter range of interest in this paper considering ${ }^{87} \mathrm{Rb}$ and ${ }^{85} \mathrm{Rb}$. 


\section{FIDELITY FREEZE FOR THE TWO-MODE BOSE-HUBBARD MODEL}

The fidelity amplitude is the overlap of the timeevolution of an initial state under a reference interaction $\hat{H}_{0}$ with the evolution of the same initial state under a slightly different Hamiltonian $\hat{H}=\hat{H}_{0}+\lambda \hat{V}$ [20]:

$$
f(t)=\left\langle\Psi_{0}\left|\hat{U}_{0}(-t) \hat{U}_{\lambda}(t)\right| \Psi_{0}\right\rangle .
$$

Here, $\left|\Psi_{0}\right\rangle$ is the initial state under consideration, $\hat{U}_{0}(t)=\hat{T} \exp \left[-\mathrm{i} \hat{H}_{0} t / \hbar\right]$ is the (time-ordered) unitary time-evolution associated to the reference Hamiltonian, $\hat{U}_{\lambda}(t)$ is the corresponding time-evolution of the perturbed Hamiltonian, and the perturbation strength is denoted formally by $\lambda$. The modulus squared of the fidelity amplitude, $F(t)=|f(t)|^{2}$, is the fidelity or Loschmidt echo [18, 19. Clearly, $F(t)$ is a measure of the sensitivity of the time evolution of $\left|\Psi_{0}\right\rangle$ to system perturbations. Another interpretation is that of an echo: $\left|\Psi_{0}\right\rangle$ evolves under $\hat{H}_{0}$ up to time $t$, when the system is suddenly reversed with respect to time, and then evolves under the action of $\hat{H}$; the Loschmidt echo compares the whole evolution with the initial state, thus quantifying the degree of irreversibility of the system. The operator $\hat{M}_{\lambda}(t)=\hat{U}_{\lambda}(-t) \hat{U}_{0}(t)$ is referred as the echo operator.

We consider the generalized Bose-Hubbard model $\hat{H}_{\mathrm{BH}}=\hat{H}_{0}+\hat{V}$ defined by

$$
\begin{gathered}
\hat{H}_{0}=\epsilon_{1} \hat{n}_{1}+\epsilon_{2} \hat{n}_{2}+\frac{U}{2}\left[\hat{n}_{1}\left(\hat{n}_{1}-1\right)+\hat{n}_{2}\left(\hat{n}_{2}-1\right)\right], \\
\hat{V}=-J_{1}\left(\hat{b}_{1}^{\dagger} \hat{b}_{2}+\hat{b}_{2}^{\dagger} \hat{b}_{1}\right)-\frac{J_{2}}{2}\left[\left(\hat{b}_{1}^{\dagger}\right)^{2} \hat{b}_{2}^{2}+\left(\hat{b}_{2}^{\dagger}\right)^{2} \hat{b}_{1}^{2}\right] .
\end{gathered}
$$

As usual, $\hat{n}_{i}=\hat{b}_{i}^{\dagger} \hat{b}_{i}(i=1,2)$ is the particle number operator of the $i$ th-mode, with $\hat{b}_{i}^{\dagger}$ and $\hat{b}_{i}$ the corresponding bosonic creation and annihilation operators, respectively. The single-particle energies of each mode are denoted by $\epsilon_{i}, U$ is the two-particle on-site interaction, $J_{1}$ is the energy of the usual (one-particle) Josephson tunneling or mode-exchange term, and $J_{2}$ is the energy associated to two-particle tunneling processes that we probe here. The total number of particles $n=n_{1}+n_{2}$ is a conserved quantity; fixing $n$, the Hilbert-space dimension is simply $n+1$. The Hamiltonian $\hat{H}_{\mathrm{BH}}$ defined through Eqs. (2) and (3) is a generalization of the usual two-mode approximation used to describe the bosonic Josephson junction [3, 4]. Below we use $J_{1}$ and $J_{2}$ as perturbation parameters, replacing $\lambda$ in Eq. (1).

We are interested in the so-called Fock regime $(U \gg$ $J_{1}>J_{2}$ ) because the fidelity freeze can be observed there. We choose the Fock (occupation-number) basis defined by $\left|\mu_{1}, \mu_{2}\right\rangle=\left(\mu_{1} ! \mu_{2} !\right)^{-1 / 2}\left(\hat{b}_{1}^{\dagger}\right)^{\mu_{1}}\left(\hat{b}_{2}^{\dagger}\right)^{\mu_{2}}|0\rangle$, where $|0\rangle$ is the vacuum state; since $n=\mu_{1}+\mu_{2}$ is conserved, we use the short-hand notation $\left|\mu_{1}\right\rangle \equiv\left|\mu_{1}, \mu_{2}\right\rangle$. By definition, $\hat{H}_{0}$ is diagonal in the Fock basis and $\hat{V}$ has vanishing diagonal matrix elements. Then, considering $\hat{H}_{0}$ as the reference interaction and $\hat{V}$ as the perturbation or residual interaction, the conditions to observe the fidelity freeze are fulfilled 25. The unperturbed spectrum is given by $E_{\mu}=E_{0}+\mu\left(\epsilon_{1}-\epsilon_{2}-U n\right)+\mu^{2} U$ with $E_{0}=\epsilon_{2} n+U n(n-1) / 2$, where $\mu=0, \ldots n+1$ labels the Fock states by mode occupation; notice the parabolic shape of $E_{\mu}$ in terms of $\mu$ for non-vanishing $U$. As it is often done we use the Heisenberg time $t_{H}=2 \pi \hbar / \bar{d}$ as the unit of time, where $\bar{d}$ is the average level spacing of the unperturbed Hamiltonian $\hat{H}_{0}$.

We compute the fidelity decay by noting that $\hat{M}_{\lambda}(t)$ is the time-evolution propagator associated with the timedependent Hamiltonian $V_{\mathrm{I}}(t)=\hat{U}_{0}(-t) \hat{V} \hat{U}_{0}(t)$ in the interaction picture [28. We use Dyson's series on the perturbation parameters $J_{r}(r=1,2)$ truncated to the second order [28, 29]. This approach is called linear response theory.

We write the fidelity amplitude as $f(t)=1+f_{1}+f_{2}+$ $\mathcal{O}\left(J_{i}^{3}\right)$, where the first- and second-order corrections (in both $J_{1}$ and $J_{2}$ ) read

$$
\begin{aligned}
f_{1} & =\sum_{r} \sum_{\mu, \nu} A_{\mu}^{*} A_{\nu} V_{\mu, \nu}^{(r)} \mathcal{I}_{1}\left[t ; \Omega_{\mu, \nu}\right] \\
f_{2} & =\sum_{r, s} \sum_{\mu, \nu, \rho} A_{\mu}^{*} A_{\nu} V_{\mu, \rho}^{(r)} V_{\rho, \nu}^{(s)} \mathcal{I}_{2}\left[t ; \Omega_{\mu, \rho}, \Omega_{\rho, \nu}\right] .
\end{aligned}
$$

Here, the matrix elements of the perturbation in the interaction picture are $\left\langle\mu\left|\hat{V}_{\mathrm{I}}(t)\right| \nu\right\rangle=\sum_{r} V_{\mu, \nu}^{(r)} \exp \left[\mathrm{i} \Omega_{\mu, \nu} t\right]$ with $\hbar \Omega_{\mu, \nu}=E_{\mu}-E_{\nu}$, and $A_{\mu}$ are the expansion coefficients of the initial state in the Fock basis. In Eqs. (4) and (5), greek letters represent the basis states and $r, s=1,2$ stand for the one- or two-particle tunneling terms of $\hat{H}_{\mathrm{BH}}$. These matrix elements read

$$
V_{\mu, \nu}^{(r)}=J_{r}\left\langle\mu\left|V^{(r)}\right| \nu\right\rangle=J_{r}\left(g_{\mu, n-\nu}^{(r)} \delta_{\mu-r, \nu}+g_{n-\mu, \nu}^{(r)} \delta_{\mu, \nu-r}\right),
$$

where $g_{\mu, \nu}^{(r)}=\left[\left(\begin{array}{l}\mu \\ r\end{array}\right)\left(\begin{array}{l}\nu \\ r\end{array}\right)\right]^{1 / 2}$. To second order in the perturbations, the fidelity is

$$
F(t)=1+2 \Re\left(f_{1}\right)+2 \Re\left(f_{2}\right)+\left|f_{1}\right|^{2} .
$$

The time dependence of Eqs. (4) and (5) appears in the (time-ordered) integrals $\mathcal{I}_{p}\left[t ; \Omega_{1}, \ldots, \Omega_{p}\right]$, where $p$ stands for the order in the Dyson's series. These integrals can be expressed recursively as

$$
\begin{aligned}
& \mathcal{I}_{p+1}\left[t ; \Omega_{1}, \ldots, \Omega_{p+1}\right]= \\
& \quad=-\frac{\mathrm{i}}{\hbar} \int_{0}^{t} \mathrm{~d} t_{1} \exp \left[\mathrm{i} \Omega_{1} t_{1}\right] \mathcal{I}_{p}\left[t_{1} ; \Omega_{2}, \ldots, \Omega_{p+1}\right],
\end{aligned}
$$

where $\mathcal{I}_{0}[t]=1$ defines the initial value of the recursion. These integrals produce terms that oscillate in time as long as the frequencies $\Omega_{\mu, \nu}$ appearing in the exponentials do not vanish, i.e. when the unperturbed spectrum is non-degenerate. Yet, certain frequency combinations may vanish and yield secular terms which grow at least linearly in time. We assume that the unperturbed spectrum is non-degenerate, which can be assured 
by choosing properly the energy difference of the two modes $\Delta \epsilon=\epsilon_{2}-\epsilon_{1}$. Then, without the secular contributions, to second-order the fidelity displays quasi-periodic oscillations in time; this is the freeze of the fidelity. The freeze of the fidelity lasts as long as the second-order approximation is valid; eventually, higher-order contributions dominate the evolution and secular terms appear that destroy the freeze of the fidelity.

Equation (7) is valid for any initial state. We consider as the initial state a normalized macroscopic trial state of the form 27 ]

$$
\begin{aligned}
\left|\Psi_{0}\right\rangle & =\left(\alpha \hat{b}_{1}^{\dagger}+\beta \mathrm{e}^{\mathrm{i} \phi} \hat{b}_{2}^{\dagger}\right)^{n}|0\rangle \\
& =\sum_{\mu}\left(\begin{array}{l}
n \\
\mu
\end{array}\right)^{1 / 2} \alpha^{\mu} \beta^{n-\mu} \mathrm{e}^{\mathrm{i}(n-\mu) \phi}|\mu\rangle .
\end{aligned}
$$

This initial state is coherent [27]; with $\alpha=\left(n_{1} / n\right)^{1 / 2}$ and $\beta=\left(n_{2} / n\right)^{1 / 2}$, it corresponds to the mean-field state having $n_{1}$ particles in the first mode and $n_{2}=n-n_{1}$ in the second one.

Inserting Eqs. (6) and (9) in (4) and (5), we obtain

$$
\begin{aligned}
f_{1} & =\sum_{r} J_{r} \sum_{\mu, \nu} A_{\mu}^{*} A_{\nu} \mathcal{I}_{1}\left[t ; \Omega_{\mu, \nu}\right] \\
& \times\left[g_{\mu, n-\nu}^{(r)} \delta_{\nu, \mu-r}+g_{n-\mu, \nu}^{(r)} \delta_{\nu, \mu+r}\right] \\
f_{2}= & \sum_{r, s} J_{r} J_{s} \sum_{\mu, \nu, \rho} A_{\mu}^{*} A_{\nu} \mathcal{I}_{2}\left[t ; \Omega_{\mu, \rho}, \Omega_{\rho, \nu}\right] \\
\times & {\left[g_{\mu, n-\rho}^{(r)} \delta_{\rho, \mu-r}\left(g_{\rho, n-\nu}^{(s)} \delta_{\nu, \rho-s}+g_{n-\rho, \nu}^{(s)} \delta_{\nu, \rho-s}\right)\right.} \\
+ & \left.g_{n-\mu, \rho}^{(r)} \delta_{\rho, \mu+r}\left(g_{\rho, n-\nu}^{(s)} \delta_{\nu, \rho-s}+g_{n-\rho, \nu}^{(s)} \delta_{\nu, \rho+s}\right)\right] .
\end{aligned}
$$

The time dependence can be further described by noting that $\mathcal{I}_{1}[t ; \Omega]=(1-\exp (\mathrm{i} \Omega t)) / \hbar \Omega$, and $\mathcal{I}_{2}\left[t ; \Omega_{1}, \Omega_{2}\right]=$ $\left(\mathcal{I}_{1}\left[t ; \Omega_{1}\right]-\mathcal{I}_{1}\left[t ; \Omega_{1}+\Omega_{2}\right]\right) / \hbar \Omega_{2}$, for $\Omega_{1}$ and $\Omega_{2}$ non-zero. These conditions are fulfilled by the assumption of a nondegenerate spectrum. Yet, for $\Omega_{1}+\Omega_{2}=0$ a secular term is obtained for $f_{2}$ which has the form $-\mathrm{i} t / \hbar$. This term does not affect the fidelity according to Eq. (7), since it is purely imaginary. Then, to second-order in the tunneling rates, the time-dependence of the fidelity is at most quasiperiodic, hence fidelity exhibits a freeze. The time during which the fidelity freeze lasts scales as the inverse of the perturbation and the inverse of $n$; see [29].

In order to obtain the fidelity freeze we extract the time-independent contributions of the integrals (8) in the expression for $\Re\left(f_{1}\right), \Re\left(f_{2}\right)$ and $\left|f_{1}\right|^{2}$. Including the dependency of $A_{\mu}$ on the phase $\phi$, cf. Eq. (9), we obtain

$$
\begin{aligned}
& \Re\left[\mathrm{e}^{\mathrm{i} p \phi} \mathcal{I}_{1}[t ; \Omega]\right] \rightsquigarrow \frac{\cos (p \phi)}{\hbar \Omega}, \\
& \Re\left[\mathrm{e}^{\mathrm{i} p \phi} \mathcal{I}_{2}\left[t ; \Omega_{1}, \Omega_{2}\right]\right] \rightsquigarrow \frac{\cos (p \phi)}{\hbar^{2} \Omega_{2}}\left(\frac{1}{\Omega_{1}}-\frac{1-\delta_{1,-2}}{\Omega_{1}+\Omega_{2}}\right), \\
& \Re\left[\mathrm{e}^{\mathrm{i} p \phi} \mathcal{I}_{1}^{*}\left[t ; \Omega_{1}\right] \mathcal{I}_{1}\left[t ; \Omega_{2}\right]\right] \rightsquigarrow \frac{\cos (p \phi)}{\hbar^{2} \Omega_{1} \Omega_{2}}\left(1+\delta_{1,2}\right) .
\end{aligned}
$$

Here, the right-hand side of these expressions are the time-independent contributions, where $p$ is an integer related to the indexes of the Fock states involved, and we have used the Kronecker-delta $\delta_{1,-2}$ to indicate that the frequencies satisfy $\Omega_{1}=-\Omega_{2}$ (indexes are reversed), and $\delta_{1,2}$ to denote that $\Omega_{1}=\Omega_{2}$ (indexes are the identical). Note that in Eq. (13) the secular term related to $\Omega_{1}+\Omega_{2}=0$ is not included due to the $\delta_{1,-2}$.

Inserting Eqs. (10) and (11) into (7), and using the time-independent contributions, Eqs. (12) to (14), we obtain

$$
\begin{aligned}
F_{\mathrm{Fr}}=1+ & 2 \sum_{r, \mu} J_{r} \frac{\left|A_{\mu}^{*} A_{\mu \pm r}\right| \mathcal{G}_{\mu}^{( \pm r)} \cos (r \phi)}{\hbar \Omega_{\mu, \mu \pm r}}+2 \sum_{r, s, \mu} J_{r} J_{s} \frac{\left|A_{\mu}^{*} A_{\mu \pm r}\right| \mathcal{G}_{\mu}^{( \pm r)} \mathcal{G}_{\mu \pm r}^{( \pm s)} \cos ((\mp r \mp s) \phi)}{\hbar^{2} \Omega_{\mu \pm r, \mu \pm r \pm s}}\left(\frac{1}{\Omega_{\mu, \mu \pm r}}-\frac{1-\delta_{ \pm r, \mp s}}{\Omega_{\mu, \mu \pm r \pm s}}\right) \\
& +\sum_{r, s, \mu, \nu} J_{r} J_{s} \frac{\left|A_{\mu}^{*} A_{\mu \pm r}\right|\left|A_{\nu}^{*} A_{\nu \pm s}\right| \mathcal{G}_{\mu}^{( \pm r)} \mathcal{G}_{\nu \pm r}^{( \pm s)} \cos ((\mp r \pm s) \phi)}{\hbar^{2} \Omega_{\mu, \mu \pm r} \Omega_{\nu, \nu \pm s}}\left(1+\delta_{ \pm r, \pm s}\right)
\end{aligned}
$$

The coefficients $\mathcal{G}_{\mu}^{(-r)}=g_{\mu, n-\mu+r}^{(r)}$ and $\mathcal{G}_{\mu}^{(+r)}=g_{n-\mu, \mu+r}^{(r)}$ are introduced to have a more compact expression. The signs of $r$ and $s$ are independent and correspond to the distinct possibilities imposed by the Kronecker deltas that appear in Eqs. (10) and (11). Equation (15) is a central result of this paper.

In Fig. 1 we show an example of the decay of fidelity for a coherent state with $n_{1}=n_{2}=n / 2$ and $\phi=\pi / 4$ obtained numerically. The figure illustrates the oscillations during the freeze of the fidelity, the eventual decay, and the value obtained from Eq. 15 for the freeze of the fidelity (horizontal green line). Time is measured in Heisenberg-time units $t_{H}$. The parameters of the model are $U=1, J_{1}=10^{-6}, J_{2}=10^{-8}, \epsilon_{1}=0.76, \epsilon_{2}=0.93$ and $n=128$; the values of $\epsilon_{i}$ assure the non-degeneracy of the spectrum of $\hat{H}_{0}$ (see below). These parameters have been chosen to simplify the numerics; other values display qualitative similar behavior as long as we are in the Fock regime. In the inset we display the result considering the second-order expansion (7); the value of $F_{\mathrm{Fr}}$ is 


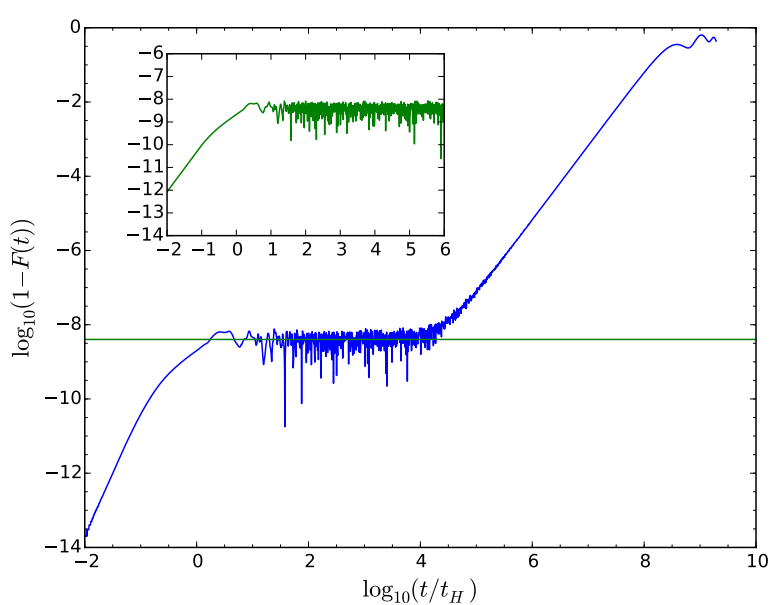

FIG. 1: (Color online) Time-dependence (in Heisenberg time units) of the fidelity obtained numerically for the extended Bose-Hubbard model in log-log scale, using an initial coherent state Eq. (9) with $n_{1}=n_{2}=n / 2=64$ and $\phi=\pi / 4$. The parameters for the Hamiltonian are $U=1, J_{1}=10^{-6}$, $J_{2}=10^{-8}, \epsilon_{1}=0.76$ and $\epsilon_{2}=0.93$. The horizontal line corresponds to the value of the fidelity freeze $F_{\text {Fr }}$ obtained from Eq. (15). In the inset we present the result of the second-order linear-response theory.

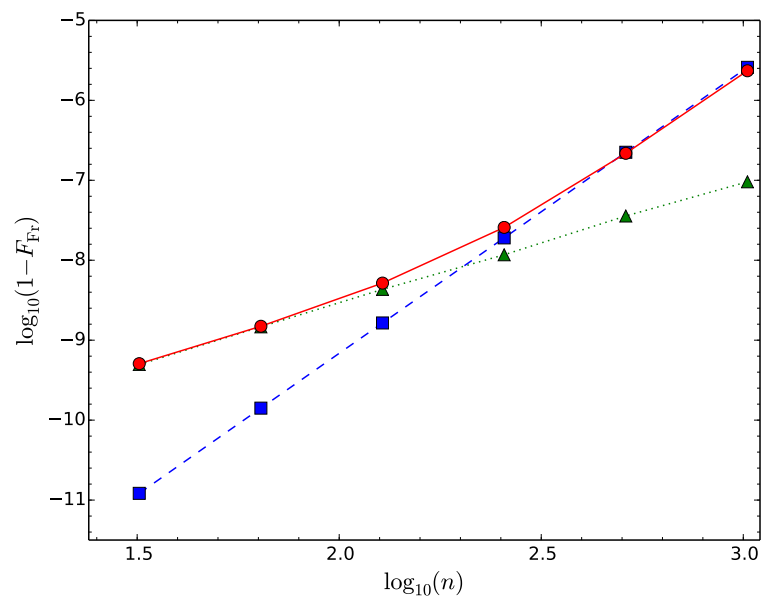

FIG. 2: (Color online) Log-log plot of $1-F_{\mathrm{Fr}}$ as a function of the number of particles, for an initial symmetric coherent state $n_{1}=n_{2}=n / 2$. Triangles (green, dotted line) correspond to the parameters $J_{1}=10^{-6}$ and $J_{2}=0$, squares (blue, dashed line) to $J_{1}=0$ and $J_{2}=10^{-8}$, and circles (red, continuous line) to $J_{1}=10^{-6}$ and $J_{2}=10^{-8}$; the remaining parameters are those of Fig. 1 .

an average over the quasi-periodic oscillations that take place during the freeze.

\section{SCALING PROPERTIES OF THE FIDELITY FREEZE}

We address now the scaling of $F_{\mathrm{Fr}}$ in terms of the number of particles. An estimate of the scaling properties is obtained considering the maximum contribution of the $n$-dependent terms in Eq. (15). This follows from a Fock state that we write as $\mu=\lambda n$, and then use Stirling's formula for large $n$. It can be shown that $\left|A_{\mu}^{*} A_{\mu \pm r}\right| \sim n^{1 / 2}$ and $\mathcal{G}_{\mu \pm r}^{( \pm s)} \sim n^{s}$ for $\lambda=\alpha^{2}$. The scaling laws of the timeindependent contributions thus read $\Re\left(f_{1}\right) \sim J_{r} n^{r-1 / 2}$, $\Re\left(f_{2}\right) \sim J_{r}^{2} n^{2 r-1 / 2}$ and $\Re\left(\left|f_{1}\right|^{2}\right) \sim J_{r}^{2} n^{2 r-1}$. Hence, the asymptotic dominating contribution for the fidelity freeze scales as

$$
1-F_{\mathrm{Fr}} \sim J_{r}^{2} n^{2 r-1 / 2} .
$$

This result predicts a different scaling for each of the tunneling terms $J_{1}$ and $J_{2}$. Thus, $F_{\mathrm{Fr}}$ exhibits a transition from a behavior dominated by $J_{1}$ to a regime where $J_{2}$ dominates, around $n \sim J_{1} / J_{2}$. Figure 2 is the numerical confirmation of this statement. The data points were obtained numerically from the time series (cf. Fig. 1), using the maxima of the quasi-periodic oscillations of $1-F(t)$ during the freeze; these values underestimate the theoretical expectation for $F_{\mathrm{Fr}}$. Fitting the data to straight-lines when either $J_{2}$ or $J_{1}$ are absent yields the slopes 1.52 and 3.54, respectively. These values are in excellent agreement with the $3 / 2$ and $7 / 2$ predicted by Eq. (16), thus showing that the scaling properties of the fidelity freeze in terms of $n$ probe the presence of twoparticle tunneling processes. Equation (16) remains valid for small departures from the symmetric initial coherent states, i.e., $\lambda \sim \alpha^{2}$.

An important assumption that we made in the derivation of Eqs. 15 and 16 is that the spectrum of $\hat{H}_{0}$ is non-degenerate, which can be fulfilled by tuning $\Delta \epsilon$, the energy difference of the two modes. As we approach a degeneracy, the appearance of secular terms makes Eq. (15) no longer valid. This can be exploited to probe the relevance of two-mode exchange processes.

To clarify this idea we consider the Fock state $\mu_{0}=$ $\lfloor(n+\Delta \epsilon / U) / 2\rceil$ whose energy is the minimum of the spectrum of $\hat{H}_{0}$, where $\lfloor x\rceil$ is the round-to-nearest integer function; note that this minimum corresponds to the parabolic shape of $E_{\mu}$ induced by a non-vanishing $U$. Assuming that $n$ is even for concreteness, it can be shown that $\Delta \epsilon / U=0$ implies that $E_{\mu_{0}-1}=E_{\mu_{0}+1}$, meaning that the Fock states $\mu_{0}-1$ and $\mu_{0}+1$ are degenerate; these states are coupled by a two-mode tunneling term. The same holds for $\Delta \epsilon / U=2$, though the actual value of $\mu_{0}$ has changed. For $\Delta \epsilon / U=1$ we have $E_{\mu_{0}}=E_{\mu_{0}+1}$, i.e. the ground state is degenerate, which also holds for $\Delta \epsilon / U=3$; in this case, the states are coupled by a one-particle tunneling term. Then, by tuning the single-particle energies, as we approach $\Delta \epsilon / U= \pm 1$ or \pm 3 , a peak in $\log _{10}\left(1-F_{\mathrm{Fr}}\right)$ develops indicating that the perturbation does contain a one-particle tunneling 


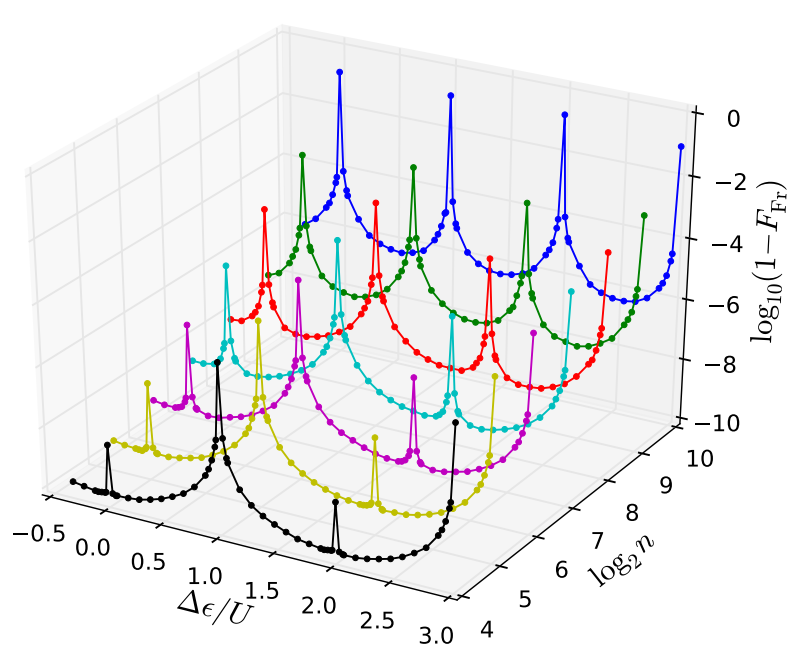

FIG. 3: (Color online) Behavior of $\log _{10}\left(1-F_{F r}\right)$ as a function of the energy difference between modes $\Delta \epsilon$ scaled by the two-particle interaction coefficient $U$ of the Bose-Hubbard model, Eq. 2 The 3D plot depict the appearance of a peak around $\Delta \epsilon / U=0,2$ which becomes noticeable as the particle number $n$ increases.

term; likewise, a peak at $\Delta \epsilon / U=0$ and \pm 2 appears if there are two-particle tunneling processes. This is illustrated in Fig. 3. which depicts $\log _{10}\left(1-F_{\mathrm{Fr}}\right)$ in terms of $\Delta \epsilon / U$ for various even values of $n$. Note that the narrow peaks at $\Delta \epsilon / U=0, \pm 2$, the signature of the two-particle tunneling, grow for increasing values of $n$. For odd values of $n$ the same argument applies, exchanging only the location of the peaks. Thus, by increasing $n$, the peaks associated with the two-particle tunneling processes become comparable to those associated to the one-particle tunneling processes; for big enough $n$ the distance between prominent neighboring peaks is halved. This result means that the fidelity freeze $F_{\mathrm{Fr}}$ can also be maximized by tuning $\Delta \epsilon / U$.

\section{SUMMARY AND OUTLOOK}

Summarizing, we have found that the fidelity freeze from an initial symmetric coherent state is a sensitive quantity to two particle mode-exchange processes in the Bose-Hubbard model. This sensitivity can be controlled with two experimental parameters: the total atom number $n$ and the energy difference between modes $\Delta \epsilon / U$. In terms of $n$, the fidelity freeze displays a transition from a regime dominated by the one-particle exchange term, for small particle numbers, to the dominance of two-particle tunneling processes when $n$ is large enough $\left(n \sim J_{1} / J_{2}\right)$. There, the fidelity freeze can also be maximized by tuning $\Delta \epsilon / U$.

Our findings hold in the Fock regime of a double well potential, i.e. for $J_{r} / U \ll 1$. Their test would face two technological challenges: measuring fidelity and produc-

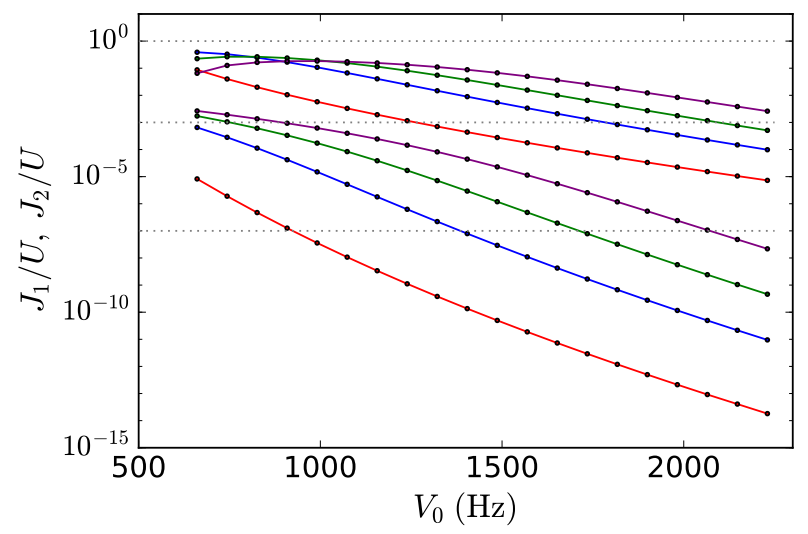

FIG. 4: Computed tunneling parameters of the two-mode BEC model as a function of the potential-barrier height, where the trap considered corresponds to that of Ref. 8. The red curves correspond to ${ }^{87} \mathrm{Rb}$, and the blue, green and purple to ${ }^{85} \mathrm{Rb}$ with s-wave scattering lengths of $20 a, 50 a$ and $100 a$, respectively, where $a=5.32 \mathrm{~nm}$ is the s-wave scattering length of ${ }^{87} \mathrm{Rb}$. The upper curves correspond to $J_{1} / U$ and the lower to $J_{2} / U$.

ing a BEC with an adequate atom number and confining geometry. Measuring fidelity is not a simple task though it has been achieved in NMR polarization echospin experiments [30, 31] and in periodically-kicked cold atoms 32. Echo spectroscopy experiments in cold atoms by Andersen et al. 33. demonstrated measurements of a quantum fidelity defined differently; theoretical aspects of that definition are discussed in Ref. 34. Fidelity has not yet been measured in two-mode Bose-Einstein condensates, though it has been proposed in the context of cold optical lattices Ref. 35.

Regarding what is an adequate BEC species to probe our results, special care is required for having a long enough confinement with $J_{2}$ big enough, so the effects addressed here can be observed experimentally. As an example, consider ${ }^{87} \mathrm{Rb}$ using the same trap frequencies and separation of the wells as in Ref. [8], and vary the potential height in order to reach the Fock regime. In this case, the Heisenberg time is $\sim 5-7 \mathrm{~ms}$ for 1500 bosons. The duration of the fidelity freeze is $t_{H} \times 10^{4}$ (Fig. 1), which is well inside normal experimental times for Bose-Einstein condensates under ultra-high vacuum.

In Fig. 4. we present the parameters $J_{1} / U$ (upper curves) and $J_{2} / U$ (lower curve) using the formulae of Ref. [17, as a function of the barrier height $V_{0}$ for $n=$ 1500 bosons. These results were obtained by integrating the non-polynomial non-linear Schrödinger equation 36. using the standard split-slit Fourier method 37, 38. The results show that for ${ }^{87} \mathrm{Rb}$ (red curves), the two-particle tunneling parameter $J_{2} / U$ is perhaps too small to yield any measurable signal. For instance, for $V_{0} \simeq 1500 \mathrm{~Hz}$, where $J_{1} / U \sim 10^{-4}-10^{-3}$, we obtain $J_{2} / U \sim 10^{-11}$. Increasing further the potential height makes $J_{2} / U$ to decrease even further.

A more promising possibility is to consider other mech- 
anisms that increase $J_{2} / U$, e.g. approaching a Feshbach resonance; an obvious candidate is ${ }^{85} \mathrm{Rb}$ [39]. Considering the same parameters for the trap used above, the blue, green and purple curves in Fig. 4 correspond to s-wave scattering length values of $20 a, 50 a$ and $100 a$, respectively. Here we use $a=5.32 \mathrm{~nm}$, the s-save scattering length of ${ }^{87} \mathrm{Rb}$, as unit to ease the comparisons. The results show that larger values of $J_{2} / U$ are obtained and, in that sense, may be accessible to experimental observation. Yet, we note that increasing the s-wave scattering length by such amounts leads to three-body collisions which have not been taken into account in our calculations.

Double-well potentials are now a common scenario for atom interferometry with matter waves. Our findings could be useful to study coherence and decoherence effects in this context. For instance, they could help to minimize decoherence on interferometers using dense atomic clouds, where non-linearities due to collisions can be exploited to improve their accuracy [6], or give rise to optimal methods for analyzing the interference fringes imprinted by small energy differences between matter waves [15].

We are thankful to Wolf von Klitzing for discussions and encouragement. We acknowledge financial support from DGAPA-PAPIIT (UNAM) projects IG-100616 and IA-103216, and from CONACyT via the National Laboratory project 232652 . LB acknowledges support from a Moshinsky fellowship (2012).
[1] D. Jaksch, C. Bruder, J. I. Cirac, C. W. Gardiner, and P. Zoller, Phys. Rev. Lett. 81, 3108 (1998).

[2] M. Greiner, O. Mandel, T. Esslinger, T. Hänsch and I. Bloch, Nature 415, 39 (2002).

[3] G.J Milburn, J. Corney, E.M. Wright and D.F. Walls, Phys. Rev. A 55, 4318 (1997).

[4] R. Gati and M.K. Oberthaler, J. Phys. B: At. Mol. Opt. Phys. 40, R61 (2007).

[5] M.R. Andrews, et al., Science 275, 637 (1997).

[6] C. Gross, T. Zibold, E. Nicklas, J. Estève and M.K. Oberthaler, Nature 464, 1165 (2010).

[7] B. P. Anderson and M. A. Kasevich, Science 282, 1686 (1998).

[8] M. Albiez, et al., Phys. Rev. Lett. 95, 010402 (2005).

[9] F. Cataliotti, et al., Science 293, 843 (2001).

[10] A. Micheli, D. Jaksch, J. I. Cirac, and P. Zoller, Phys. Rev. A 67, 013607 (2003).

[11] B. Juliá-Díaz, et al., Phys. Rev. A 86, 023615 (2012).

[12] A. Günther, S. Kraft, C. Zimmermann, and J. Fortágh, Phys. Rev. Lett. 98, 140403 (2007).

[13] T. Schumm, et al., Nat. Phys., 1, 57 (2005).

[14] G.-B. Jo, et al., Phys. Rev. Lett. 98, 030407 (2007).

[15] F. Baumgartner, et al., Phys. Rev. Lett., 105, 243003 (2010).

[16] O. Zobay, B.M. Garraway, Phys. Rev. Lett. 86, 1195 (2001).

[17] D. Ananikian and T. Bergeman, Phys. Rev. A 73, 013604 (2006); ibid 74, 039905(E) (2006).

[18] A. Peres, Phys. Rev. A 30, 1610 (1984).

[19] R.A. Jalabert and H.M. Pastawski, Phys. Rev. Lett. 86, 2490 (2001).

[20] T. Gorin, T. Prosen, T.H. Seligman, and M. Žnidarič, Phys. Rep 435, 33 (2006); Ph. Jacquod and C. Petitjean, Adv. Phys. 58, 67 (2009); A. Goussev, et al., Scholarpedia 7, 11687 (2012).

[21] J.D. Bodyfelt, M. Hiller and T. Kottos, EPL 78, 50003 (2007).

[22] G. Manfredi and P.-A. Hervieux, Phys. Rev. Lett. 100, 050405 (2008).
[23] Qiang Zheng, et al., Phys. Rev. E 80, 016214 (2009); K. Lelas and T. Ševa and H. Buljan, Phys. Rev. A 84, 063601 (2011).

[24] T. Prosen and M. Žnidarič, New J. Phys. 5, 109 (2003); Phys. Rev. Lett. 94, 044101 (2005).

[25] T. Gorin, et al., Phys. Rev. Lett. 96, 244105 (2006).

[26] G. Manfredi and P.-A. Hervieux, Phys. Rev. Lett. 97, 190404 (2006)

[27] L. Amico and V. Penna, Phys. Rev. Lett. 80, 2189 (1998); M. Holthaus and S. Stenholm, Eur. Phys. J. B 20, 451 (2001); P. Buonsante, V. Penna and A. Vezzani, Phys. Rev. A 72, 043620 (2005).

[28] T. Prosen, Phys. Rev. E 65, 036208 (2002); T. Prosen and M. Žnidarič, J. Phys A: Math Gen 35, 1455 (2002);

[29] L. Benet, S. Hernández-Quiroz and T.H. Seligman, Phys. Rev. E 83, 056216 (2011). D. Espitia, MSc thesis, UNAM (2014).

[30] P.R. Levstein, G. Usaj and H.M. Pastawski, J. Chem. Phys. 1082718 (1998).

[31] P.R. Zangara, D. Bendersky, P.R. Levstein, H.M. Pastawski, Phil. Trans. R. Soc. A. 37420150163 (2016).

[32] Saijun Wu, et al., Phys. Rev. Lett. 103034101 (2009).

[33] M. F. Andersen, A. Kaplan and N. Davidson, Phys. Rev. Lett. 90, 023001 (2003); M. F. Andersen, A. Kaplan, T. Grunzweig and N. Davidson, Phys. Rev. Lett. 97, 104102 (2006).

[34] A. Goussev and Ph. Jacquod, Phys. Rev. A 82, 052114 (2010).

[35] F.M. Cucchietti, J. Opt. Soc. Am. B 27 A30-A35 (2010).

[36] L. Salasnich, A. Parola and L. Reatto, Phys. Rev. A 65 043614 (2002).

[37] P. L. DeVries, AIP Conference Proceedings 160269 (1987).

[38] R. Gati, Bose-Einstein condensates in a single double well potential, PhD Thesis, U. Heidelberg, 2007.

[39] S.L. Cornish, N.R. Claussen, J.L. Roberts, E.A. Cornell and C.E. Wieman, Phys. Rev. Lett. 85, 1795 (2000). 